\title{
TÉCNICAS DA ESTATÍSTICA ESPACIAL NA ANÁLISE DE DADOS DE ÁREAS NO ESTUDO DA DENGUE
}

\section{SPATIAL STATISTICS TECHNIQUES IN DATA ANALYSIS OF AREAS IN THE STUDY OF DENGUE}

\author{
Leila Maria Ferreira ${ }^{1}$ \\ Thelma Sáfadi ${ }^{2}$ \\ Renato Ribeiro de Lima $^{3}$
}

Resumo: A análise espacial tem se destacado na literatura oferecendo critérios quantitativos de agrupamento e dispersão dos dados, permitindo, entre outras coisas, a determinação da dependência espacial entre observações, além da identificação de regiões em que a doença, em estudo, apresenta comportamento semelhante. Neste trabalho, foram utilizadas diferentes técnicas da estatística espacial na análise de dados de áreas, objetivando encontrar a melhor técnica de análise. Para ilustrar a metodologia, foram analisados os casos de dengue ocorridos na cidade de Lavras - MG, no período de 2007 a 2010. Esse período corresponde ao primeiro surto da doença registrado na cidade. Os resultados da pesquisa apontaram a estatística Scan Espaço-Temporal como a melhor técnica, cujas vantagens incluem a possibilidade de localizar as áreas de maior risco de surto epidêmico.

Palavras-chave: Estatística espacial; SIGs; cluster; máxima verossimilhança; dengue.

\begin{abstract}
Spatial analysis has been highlighted in the literature offering quantitative criteria for the grouping and dispersion of data, allowing, among other things, the determination of the spatial dependence among observations, as well as the identification of regions in which the disease under study presents a similar behavior. In this work, different spatial statistics techniques were used in the data analysis of areas, aiming to find the best analysis technique. To illustrate the methodology, we analyzed the cases of dengue that occurred in the city of Lavras - MG, between 2007 and 2010. This period corresponds to the first outbreak of the disease registered in the city. The results of the research pointed to the statistic Space-Temporal Scan as the best technique, whose advantages include the possibility to locate the areas of greater risk of epidemic outbreak.
\end{abstract}

Keywords: Spatial statistics; SIGs; cluster; maximum likelihood; dengue.

\section{INTRODUÇÃO}

A estatística espacial lida com problemas associados a dados distribuídos geograficamente, que incluem padrões de pontos, superfícies contínuas e dados agregados por áreas (GRIFFITH; PAELINCK, 2011).

A análise espacial fornece um grande ferramental matemático destinado a fornecer ao pesquisador a capacidade de estabelecer critérios quantitativos de agrupamento ou dispersão dos dados espaciais, determinando o grau de dependência espacial entre as observações. A técnica da estatística espacial nasce da necessidade de quantificação da dependência espacial presente num conjunto de dados geográficos, distinguindo-se das demais técnicas empregadas em análises estatísticas por

\footnotetext{
1 Doutoranda Universidade Federal de Lavras - UFLA, Brasil. E-mail: leilamaria2003@yahoo.com.br.

2 Docente da Universidade Federal de Lavras - UFLA, Brasil. E-mail: safadi@des.ufla.br.

${ }^{3}$ Docente da Universidade Federal de Lavras - UFLA, Brasil. E-mail: rrlima@des.ufla.br.
} 
considerar explicitamente a localização espacial dos dados no processo de descrição, análise ou coleta (CÂMARA et al., 2004).

Dentre as subdivisões da estatística espacial, tem-se os dados de área os quais envolvem elementos que associam o mapa geográfico a uma base de dados. O mapa geográfico é dividido por áreas, em que cada uma possui uma ou mais variáveis aleatórias que representam um valor para toda a área e não apenas o valor de um ponto específico (que chamamos de centróide). Ou seja, não é necessário se conhecer a localização exata do evento, mas um valor agregado por área (ASSUNÇÃO, 2000).

Por intermédio do mapeamento de doenças, pode-se avaliar a variação espacial de taxas de incidência de determinadas doenças, com o intuito de realizar predições de epidemias, identificar áreas de riscos, orientar ações em saúde e levantar hipóteses (LAWSON, 2001).

Um método eficaz na prevenção de doenças graves, como a dengue, é a análise da dispersão espacial dos casos. A tecnologia do Sistema de Informação Geográfica (SIG) pode ser usada como uma ferramenta de apoio para o controle da dengue, uma vez que permite aos pesquisadores identificarem áreas com maior intensidade de transmissão, a fim de que as áreas mais vulneráveis possam ser identificadas e ações de controle possam ser planejadas (BESSA JÚNIOR et al., 2013).

Por meio da estatística de varredura espacial, desenvolvida por Kulldorff e Nagarwalla (1995), pode-se identificar os prováveis clusters nos anos analisados. Método também utilizado por Hjalmars et al. (1996), Kulldorff, Feuer e Miller (1997) e Kulldorff et al. (2006), para localização de clusters no estudo relacionado com doenças que atingem a população, buscando explicar se determinada doença está distribuída uniformemente na área estudada ou se há clusters com significância estatística. Com a identificação desses clusters, medidas de controle dessas doenças se tornam mais eficazes.

Os mapas temáticos são essenciais para avaliar a evolução temporal de casos de dengue. Os mapas são sombreados de acordo com os valores das variáveis particulares, como, por exemplo, quantidade de pessoas mortas por determinada doença (MORRISON et al., 1998; LEITE; ABREU, 2009; MONDINI et al., 2005).

A estatística de varredura espaço-temporal corresponde à interação do espaço com o tempo, essa técnica visa à detecção do provável cluster durante todo o período estudado. Metodologia utilizada por Kulldorff et al. (1998), Kulldorff (2001) e Kulldorff et al. (2005), nos estudos, respectivamente, de câncer de cérebro, em Los Alamos; câncer de tireóide, entre os homens no Novo México; e surtos de rotavírus, norovírus e gripes. Essa análise é bastante importante na área da epidemiologia, pois auxilia os sistemas de vigilância de doenças, possibilitando um direcionamento para um local específico, tornando o processo mais ágil, todavia, quando se trata de epidemias, o combate tem que ser imediato. 
O método de varredura espacial é utilizado para confirmação estatística dos agrupamentos, os quais correspondem a um conjunto de áreas que apresentam um risco significativamente alto em relação a um determinado evento (BEATO FILHO et al., 2001; FLAUZINO et al., 2009).

A análise de varredura espaço-tempo tem sido amplamente utilizada em estudos relacionados à dengue. Um exemplo é a identificação da interação entre densidade populacional e a falta de água encanada como causa de surtos de dengue nos distritos em Nha Trang, Ninh Hoa e na província Kanh Hoa no centro-sul do litoral do Vietnã. Nesse estudo, a estatística de varredura espaço-temporal foi aplicada para identificar as áreas geográficas com o maior risco (SCHMIDT et al., 2011). A dinâmica de propagação de um surto de dengue em Tartagal, no norte da Argentina, foi investigada considerando o agrupamento espaço-temporal dos casos, para tal foi utilizado o teste de Knox (ROTELA et al., 2007). O teste Knox foi também usado na análise espaçotemporal dos casos de dengue em Iracoubo, Guiana Francesa (TRAN et al., 2004). Padrões espaço-temporais de hotspots foram utilizados durante epidemias de dengue na província de Chachoengsao, Tailândia (JEEFOO; TRIPATHI; SOURIS, 2011).

Os mapas obtidos pelos coeficientes de incidência representam o risco real da doença em relação ao tamanho da população. Mondini e Chiaravalloti Neto (2007) trabalharam com os casos de dengue na cidade de São José do Rio Preto, Estado de São Paulo, utilizando o cálculo dos coeficientes de incidência por ano e quadriênio, para cada um dos agrupamentos de setores censitários. Machado, Oliveira e Santos (2009) analisaram o coeficiente de incidência acumulada de dengue durante o período de 8 anos na cidade de Nova Iguaçu, Estado do Rio de Janeiro.

O Índice Global de Moran fornece um valor único para medir a associação espacial para todo o conjunto de dados de área. Uma das vantagens do uso do índice é a capacidade de verificar se as áreas geograficamente vizinhas apresentam maior semelhança do que o esperado ao acaso quanto às variáveis estudadas (TEIXEIRA; MEDRONHO, 2008). O Índice Local de Moran já é mais específico e atribui um valor para cada área, o que permite que os agrupamentos sejam identificados. $\mathrm{O}$ valor do índice não é limitado ao intervalo $(-1,1)$, fornecendo uma medida geral da associação espacial (BAILEY; GATRELL, 1995).

Analisando a dependência espacial dos dados por meio do índice global de Moran, técnica também utilizada por Teixeira e Cruz (2011) e por Teixeira e Medronho (2008) no estudo relacionado com a epidemia de dengue, consegue-se obter informações sobre áreas geograficamente vizinhas que apresentam maior semelhança quanto às variáveis estudadas.

Com intuito de identificar os agrupamentos com significância estatística da doença na área analisada, foi utilizado o Índice Local de Moran. Outros autores como Machado, Oliveira e Santos (2009) e Almeida et al. (2008) trabalharam com esse índice na cidade de Nova Iguaçu - RJ e Belo Horizonte - MG, respectivamente, detectando os 
agrupamentos relacionados com a epidemia de dengue.

Este estudo utiliza técnicas para mapear a dengue que têm sido utilizadas para o mapeamento de doenças infecciosas desde o final dos anos 1990 (ROJAS; BARCELLOS; PEITER, 1999). O objetivo foi analisar várias técnicas da estatística espacial no estudo da ocorrência de dengue em bairros da cidade de Lavras, Minas Gerais, cujos dados correspondem a dados de áreas (devido não se ter a localização exata de cada um dos casos de dengue), em busca de se encontrar a melhor técnica de análise da estatística espacial.

\section{MATERIAL E MÉTODOS}

O Índice Global de Moran é uma das ferramentas mais utilizadas para calcular a autocorrelação espacial, esse indicador visa a identificar a estrutura de correlação espacial que melhor descreva os dados (CÂMARA et al., 2004).

Uma vez calculado o Índice Global de Moran, é importante estabelecer sua validade estatística, para verificar se os valores medidos apresentam autocorrelação espacial significativa. Valores próximos de zero indicam a inexistência de autocorrelação espacial significativa entre os valores dos objetos e seus vizinhos. Valores positivos indicam autocorrelação espacial positiva, o valor do atributo de um objeto tende a ser semelhante aos valores dos seus vizinhos. Valores negativos indicam autocorrelação espacial negativa. Para verificar a significância do índice, necessita-se associar a este uma distribuição estatística, sendo mais usual relacionar a estatística de teste à distribuição normal (BAILEY; GATRELL, 1995).

O Índice Global de Moran é definido por

$$
I=\frac{\sum_{i=1}^{n} \sum_{j=1}^{n} w_{i j}\left(z_{i}-\bar{z}\right)\left(z_{j}-\bar{z}\right)}{\sum_{i=1}^{n}\left(z_{i}-\bar{z}\right)^{2}},
$$

em que $n$ é o número de áreas, $z_{i} 0$ valor do atributo considerado na área $i, z_{j} 0$ valor do atributo considerado na área $j, \bar{z}$ é o valor médio do atributo na região de estudo e $w_{i j}$ refere-se aos elementos da matriz normalizada de proximidade espacial.

O Índice Local de Moran (LISA), ao contrário do Índice Global de Moran, produz um valor específico para cada área, permitindo a identificação de agrupamentos com valores semelhantes (clusters) ou diferentes (outliers) e de regimes espaciais, não percebidos por meio dos resultados globais. Um indicador LISA é qualquer estatística que satisfaça a dois critérios: 1) permite a identificação de padrões de associação espacial significativa para cada área da região de estudo; e 2) constitui uma decomposição do índice global de associação espacial. Ou seja, o somatório do LISA para todas as regiões é proporcional ao indicador de autocorrelação espacial global (ANSELIN, 1995). 
A significância estatística do uso do Índice Local de Moran é computada da seguinte maneira: para cada área, calcula-se o índice local, e depois permuta-se, aleatoriamente, o valor das demais áreas, até se obter uma pseudodistribuição para a qual possamos computar os parâmetros de significância. Uma vez determinada a significância estatística do Índice Local de Moran, é útil gerar um mapa indicando as regiões que apresentam correlação local significativamente diferente das demais áreas. Essas regiões podem ser vistas como "bolsões" de não estacionariedade (áreas com dinâmica espacial própria, ou seja, sem um padrão de regularidade), que merecem análise detalhada (CÂMARA et al., 2004).

O Índice Local de Moran é dado por

$$
I_{i}=\frac{z_{i} \sum_{j=1}^{n} w_{i j} z_{i}}{\sum_{j=1}^{n} z_{j}^{2}}
$$

em que $n$ é o número de áreas, $z_{i} 0$ valor do atributo considerado na área $i, z_{j} 0$ valor do atributo considerado na área $j$, e $w_{i j}$ refere-se aos elementos da matriz normalizada de proximidade espacial.

$\mathrm{Na}$ análise puramente espacial, um cluster refere-se a uma observação de valores, no qual, em determinada(s) área(s) há uma concentração maior sem a interferência do período de tempo considerado. O modelo mais utilizado nessa análise é o de Poisson, por serem dados de contagem (LUCENA; MORAES, 2012). Essa análise utiliza o método da máxima verossimilhança que prioriza uma região formada pelas áreas cujos centróides correspondem ao centro de um círculo, e em que ocorrem a variação do raio e do centro do círculo. Em cada círculo é calculada a razão entre o máximo da verossimilhança sob a hipótese alternativa de que o número de casos dentro do círculo é maior do que fora e o máximo da verossimilhança sob a hipótese nula de que o valor encontrado é casual. Logo depois, é calculado o máximo dessas razões para todos os círculos possíveis. Esse valor máximo do teste da razão da máxima verossimilhança é designado de T e essa avaliação é realizada por simulações de Monte Carlo. A região associada com esse máximo é chamada de cluster mais verossímil ou primário (ASSUNÇÃO, 2000).

$\mathrm{Na}$ análise Espaço-Tempo, uma característica da varredura é permitir a incorporação do fator temporal no estudo. Nesse caso, em específico, é de interesse identificar clusters de doenças que venham a ocorrer no espaço e no tempo simultaneamente. O procedimento é análogo ao método espacial, sendo que, nesse caso, em vez de círculos, são utilizados cilindros, cuja base representa o espaço geográfico e a altura representa o tempo. Embora o procedimento permita a base e a altura variarem continuamente de acordo com a varredura do método no espaço e no tempo, não há alterações em relação à razão de verossimilhança e a significância do teste (KULLDORFF et al., 1998).

O modelo mais utilizado, neste caso em específico, é o de Permutação espaçotemporal, empregado por Lucena e Moraes (2012), dado por 


$$
P\left(c_{A}\right)=\frac{\left(\begin{array}{c}
\sum_{t \in \Omega} \sum_{z \in A} c_{z} \\
c_{A}
\end{array}\right)\left(\begin{array}{c}
C-\sum_{t \in \Omega} \sum_{z \in A} c_{z t} \\
\sum_{z \in \Omega} \sum_{t \in A} c_{z t}-c_{A}
\end{array}\right)}{\left(\begin{array}{c}
C \\
\sum_{t \in \Omega} \sum_{z \in A} c_{z t}
\end{array}\right)},
$$

em que $\boldsymbol{c}_{\boldsymbol{A}}$ é o número de casos observados no cilindro $A$;

$c_{z t}$ é o número de casos observados na área $Z$ durante o tempo $t$;

$\Omega=A \cup A^{c}$

Utilizando o programa livre SaTScan (versão 9.1; disponível em http://www.satscan.org), foram realizadas as análises espaço-temporal e puramente espaciais, fazendo uso da metodologia desenvolvida por Kulldorff e Nagarwalla (1995). Os dados de entrada para a análise espaço-temporal foram o número de casos de dengue e as coordenadas cartesianas dos centróides de cada bairro, que foram obtidos com 0 programa livre TerraView (version 3.4.0; disponível em http://www.dpi.inpe.br/terraview/index.php). O tipo de análise espaço-tempo realizada foi a retrospectiva e o modelo probabilístico utilizado foi o de permutação espaço-tempo. Utilizou-se o método de varredura de áreas com altas taxas e o tempo de agregação foi de um ano. $\mathrm{Na}$ análise puramente espacial, o modelo probabilístico utilizado foi o de Poisson, sendo realizada uma análise espacial para cada ano.

O modelo de Poisson é dado por

$$
L(z, \hat{p}(z), \hat{q}(z))=\frac{\exp \left[-p(z) n_{z}-q(z)\left(M-n_{z}\right)\right]}{C !} p(z)^{C_{z}} q(z)^{C-c_{z}} \prod_{i} c_{i},
$$

em que $z$ é um candidato a cluster, $p(z)$ a probabilidade do fenômeno em estudo ocorrer dentro do círculo e $q(z)$ a probabilidade do fenômeno ocorrer fora dele. $C$ é o número total de casos em toda a região do estudo e $M$ é a população total. A função exponencial é representada por $\exp , c_{z}$ e $c_{i}(i, z=1,2, \ldots, k)$ são, respectivamente, o número de casos no círculo $z$ e círculo $i$ e $n_{z}$ é o número de indivíduos em risco no círculo $z$.

Foram considerados, na análise, números de casos de dengue em 91 bairros da cidade de Lavras - MG, no período de 2007 a 2010, obtidos junto à Secretaria Municipal de Saúde. Lavras é um município pertencente à região do Sul do estado de Minas Gerais, Brasil. A cidade está localizada na latitude $21^{\circ} 14^{\prime} 30^{\prime \prime}$ Sul e longitude $44^{\circ} 00^{\prime} 10^{\prime \prime}$ Oeste.

A cidade está a 919 metros acima do nível do mar e possui uma área de 564,7 $\mathrm{km}^{2}$. Tem uma população urbana de 101.208 habitantes. O clima é classificado como tropical de altitude. (IBGE, 2017).

Na Figura 1, tem-se o mapa da cidade de Lavras com as identificações (IDs) dos 91 bairros.

As estimativas dos tamanhos das populações para cada bairro foram baseadas no número de eleitores ativos em cada setor censitário, porque essa foi a única alternativa disponível para estimar a população na cidade. O programa livre TerraView 
foi usado para gerar os mapas de incidência e temáticos e para calcular os Índices de Moran Global e Local para cada um dos anos de estudo. No processo de inferência para os Índices de Moran, 999 permutações foram geradas, que corresponde ao teste com nível de significância estatística de $1 \%$.

Figura 1. Identificações (IDs) dos bairros da cidade de Lavras. Cada número no mapa está associado ao nome do bairro.

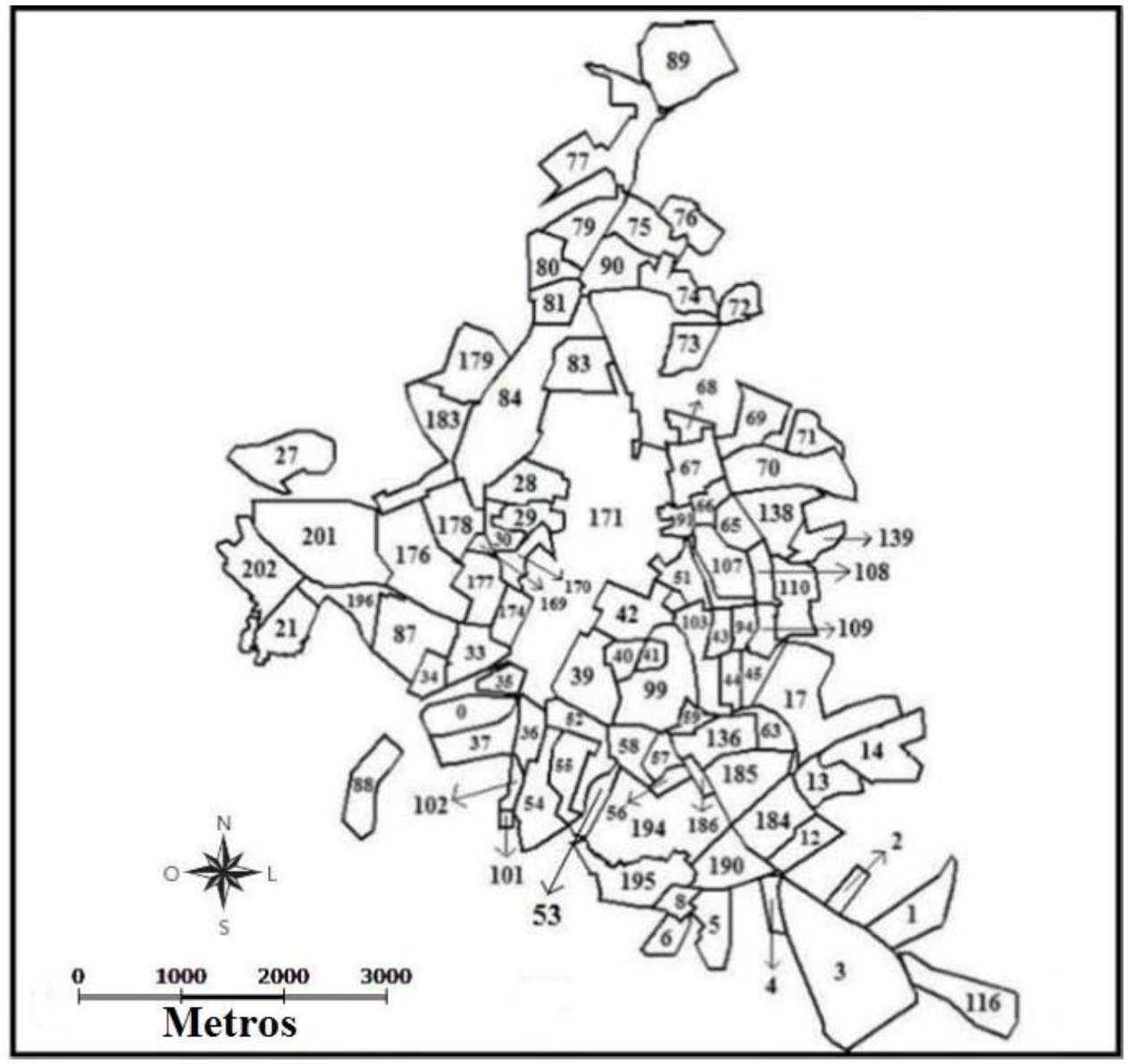

Fonte: Autores.

Para cada ano de estudo, o valor de incidência da dengue, $\mathrm{R}$, em cada bairro foi calculado como a razão entre o número de casos de dengue $(N C)$ e número de habitantes por bairro (ND) vezes 1.000. Esta técnica foi usada, anteriormente, por Machado et al. (2009). Os mapas foram gerados a partir dos valores obtidos. Uma série de incidência pode ser estudada a partir da incidência anual, o que pode revelar o comportamento da doença ao longo do tempo (MONDINI; CHIARAVALLOTI NETO, 2007).

\section{RESULTADOS E DISCUSSÃO}

O número de casos de dengue registrados na cidade de Lavras - MG, em cada ano, foram: 442 casos, em 2007, que corresponde ao primeiro surto epidêmico da doença na cidade; 77 casos em 2008, 3 casos em 2009 e em 2010, a situação torna-se 
crítica novamente, tendo 714 casos registrados - o maior número de casos na história da cidade. Durante esses quatros anos, a cidade registrou um total de 1.236 casos confirmados de dengue.

Uma análise exploratória da dengue ao longo dos anos pode ser observada na Figura 2 de (e) a (h). As áreas nas cores escuras identificam as regiões com o maior número de casos de dengue. No ano de 2007, há maior concentração na região oeste da cidade. Em 2008 na parte central e em 2010 na parte central e oeste.

Figura 2. Incidência da dengue (razão do número de casos de dengue dividido pelo número de habitantes por bairro vezes 1.000) nos anos (a) 2007, (b) 2008, (c) 2009 e (d) 2010. Casos de dengue nos anos (e) 2007, (f) 2008, (g) 2009 e (h) 2010.

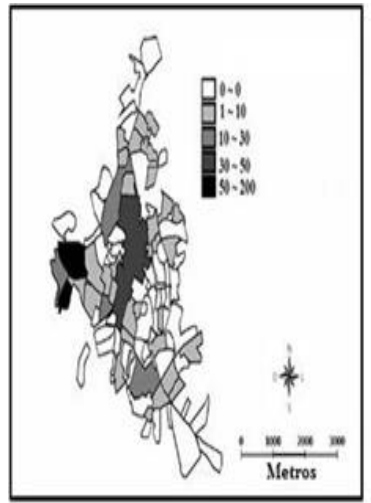

(a)

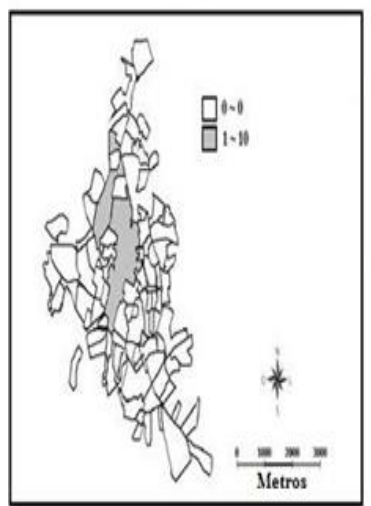

(c)

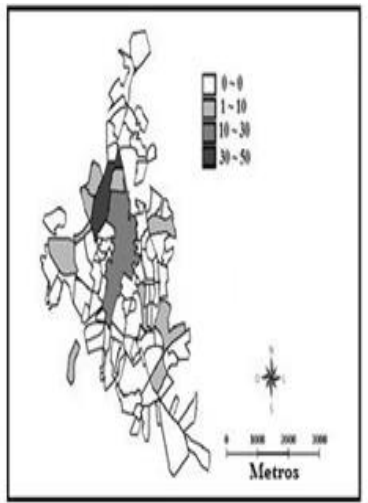

(b)

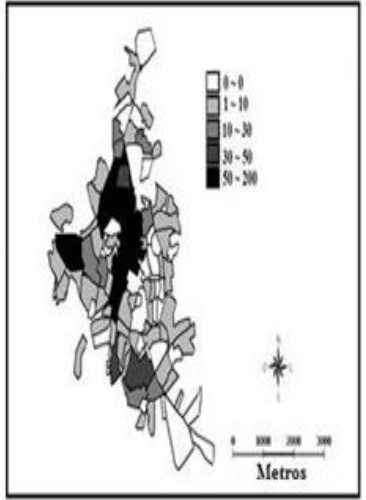

(d)

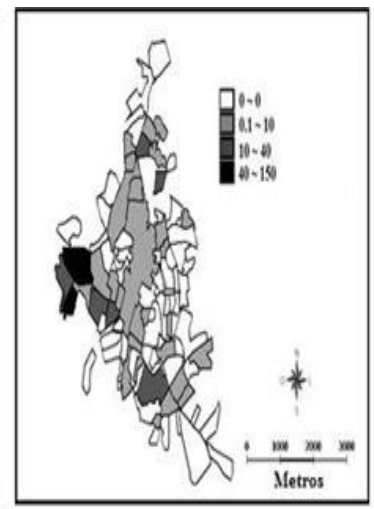

(e)

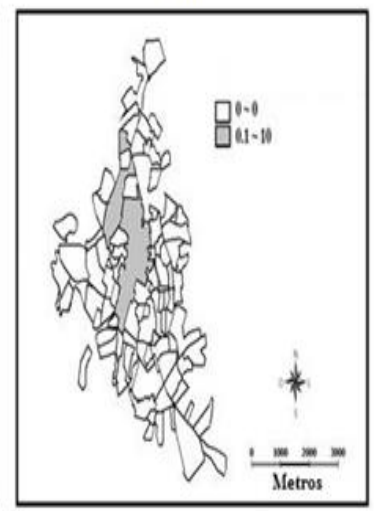

(g)

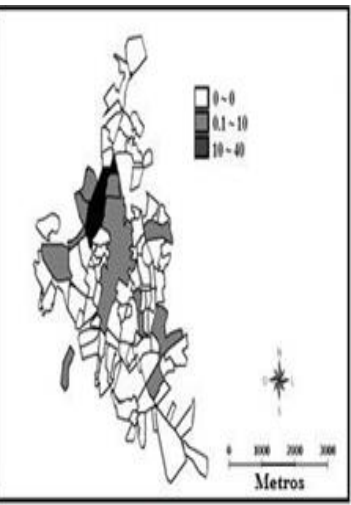

(f)

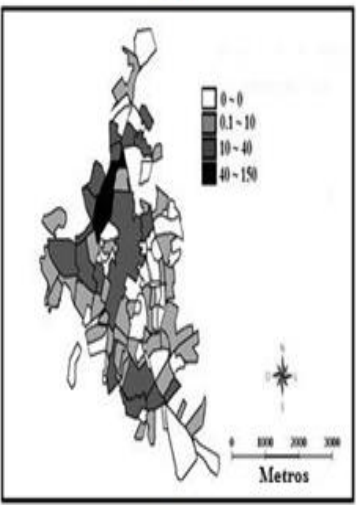

(h)

Fonte: Autores.

Por meio dos mapas temáticos, pode-se ver claramente o avanço da doença, técnica também utilizada por Galli e Chiaravalloti Neto (2008). Comparando os anos de 2007 e 2008, os bairros com o maior número de casos no ano de 2007 não registraram casos no ano de 2008. Com relação ao ano de 2008, o bairro que teve o maior registro de casos foi o de ID 84, registrando casos em 2007, 2009 e 2010. Em 2010, o bairro de ID 171 que registrou o maior número de casos, apareceu nos anos anteriores também, evidenciando a persistência da doença. Como os bairros de IDs 84 e 171 registraram casos da doença em todos os anos, medidas de erradicação da doença necessitariam ser intensificadas nesses bairros. 
Visualizando os mapas de incidência apresentados na Figura 2 de (a) a (d), é possível se ter uma visão mais detalhada sobre os bairros mais críticos, em relação ao número de casos de dengue. O cálculo dos coeficientes de incidência foi feito para cada bairro e para cada ano analisado. Dentre os coeficientes calculados, o bairro que teve a maior incidência da doença foi o de ID 21, no ano de 2007, seu coeficiente foi de 124,13 por mil habitantes, ou seja, os moradores desse bairro corriam grande risco de contraírem a doença.

Comparando os mapas de incidência com os mapas temáticos, verificou-se que nos anos de 2007, 2008 e 2009, os bairros mais críticos com relação a dengue são os mesmos. Apenas no ano de 2010 o resultado foi diferente, somente a ID 84 teve a maior taxa de incidência.

Verificou-se que nos anos 2007, 2008 e 2010, os valores do Índice Global de Moran foram, respectivamente: $0,1138,0,1074$ e 0,0917 , valores que ficaram no intervalo entre $(0,1)$, que corresponde a uma correlação direta dos dados, ou seja, existe dependência espacial. Nesses anos, os valores-p foram menores que 0,05, comprovando a existência de dependência espacial, pois rejeita-se a hipótese nula de independência espacial. No ano de 2009, o índice de Moran foi de $-0,0234$, tendo um valor-p acima de 0,05, o que indica que, nesse ano, os casos ocorreram de forma aleatória. Esse resultado era esperado, pois nesse ano ocorreu um número pequeno de casos.

O Índice Local de Moran foi calculado para cada bairro. A interpretação dos resultados segue os mesmos parâmetros do Índice Global de Moran.

Para gerar os mapas com a identificação dos clusters, de acordo com a Figura 3, foi feita uma consulta nos valores-p, e foram localizados os bairros com níveis de significância estatística menores que 0,05 , obtidos por meio do cálculo do Índice Local de Moran.

Em 2007, foram identificados 4 clusters como tendo dependência espacial. Cluster 1 inclui dois bairros na região ao norte. Cluster 2 inclui três bairros na região a oeste. Cluster 3 inclui cinco bairros na região a leste. Cluster 4 inclui quarto bairros na região sul.

Em 2008, 6 clusters foram identificados. Cluster 1, inclui dezoito bairros nas regiões norte, central e oeste. Cluster 2, inclui dois bairros na região oeste. Cluster 3 , inclui dois bairros na região central. Cluster 4, inclui seis bairros na região leste. Cluster 5 , inclui treze bairros na região central. Cluster 6 , inclui cinco bairros na região sul.

Em 2010, cluster 1 inclui quinze bairros nas regiões norte e central. Cluster2, inclui dois bairros na região leste. Cluster 3, inclui quarto bairros na região leste. Cluster 4, inclui dois bairros na região sul. 
Verifica-se que o maior número de clusters identificados correspondem ao ano de 2008, totalizando seis clusters.

Com os dados apresentados na Tabela 1, pode-se encontrar os prováveis clusters que apresentaram significância estatística, segundo 0 teste da razão de verossimilhança, com valor $p<0,05$, utilizando o programa SaTScan na análise puramente espacial.

Figura 3. Bairros com dependência espacial nos anos de (a) 2007, (b) 2008 e (c) 2010.

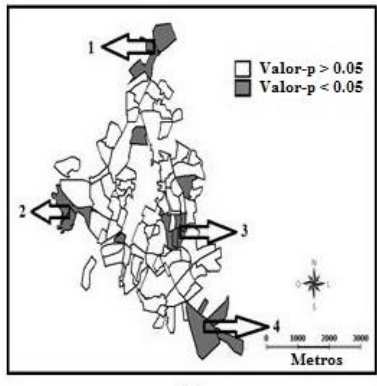

(a)

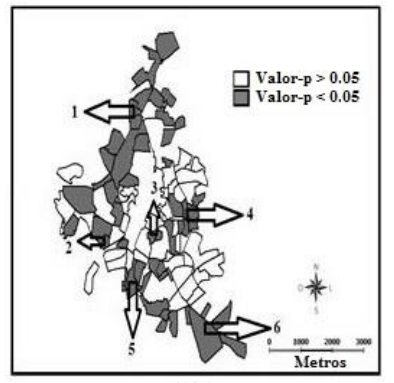

(b)

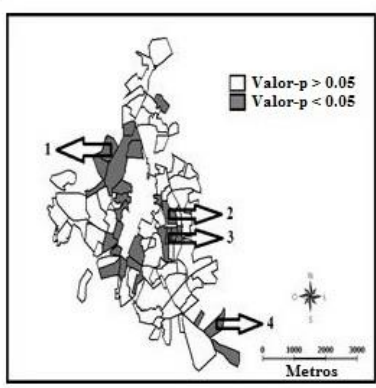

(c)

Fonte: Autores.

Os clusters identificados na análise espacial são apresentados na Figura 4. Esses resultados mostram que o cluster mais provável que foi detectado para o ano de 2007 inclui as seguintes IDs: 202, 21 e 201. Em 2008, o cluster mais provável inclui as IDs 179, 84 e 183. Em 2010, o cluster mais provável inclui as IDs 83, 81, 84, 90, 80, 73, 79, $68,74,28,79,75,67$ e 171.

O tamanho da população do cluster mais provável que foi detectado para o ano de 2007 foi de 4.294 habitantes e ocorreram 269 casos de dengue nessa região do cluster. Em 2008, o tamanho da população foi de 3.647 e ocorreram 50 casos de dengue. Em 2010, o tamanho da população foi de 23.551 e ocorreram 387 casos de dengue. 
Tabela 1. Valores da Análise Espacial.

\begin{tabular}{lccc}
\hline Ano & $\mathbf{2 0 0 7}$ & $\mathbf{2 0 0 8}$ & $\mathbf{2 0 1 0}$ \\
\hline Coordenadas & $(497476,7.65072 \mathrm{e}+006)$ & $(499090,7.65227 \mathrm{e}+006)$ & $(499944,7.65226 \mathrm{e}+006)$ \\
Raio & 569,84 & 625,78 & 1180,90 \\
População & 4294 & 3647 & 23551 \\
Número de casos & 269 & 50 & 387 \\
Casos esperados & 21,74 & 3,08 & 182,65 \\
Observado/esperado & 12,38 & 16,24 & 2,12 \\
Risco relativo & 30,06 & 44,47 & 3,44 \\
Razão de & 523,2 & 112.2 & 131,8 \\
verossimilhança & & & \\
Valor-p & $<1 \times 10^{-17}$ & $<1 \times 10^{-17}$ & $<1 \times 10^{-17}$ \\
IDs dos prováveis & $202,21,201$ & $179,84,183$ & $83,81,84,90,80,73,179$, \\
Clusters & & & $68,74,28,79,75,67,171$ \\
\hline
\end{tabular}

Fonte: Autores.

Com 14 bairros registrados, o cluster que foi detectado em 2010 teve o maior número de bairros com dependência espacial em comparação com 2007 e 2008. Como esses bairros estão próximos uns dos outros, eles apresentaram incidências semelhantes da doença.

Figura 4. Bairros com dependência especial nos anos (a) 2007, (b) 2008 e (c) 2010.

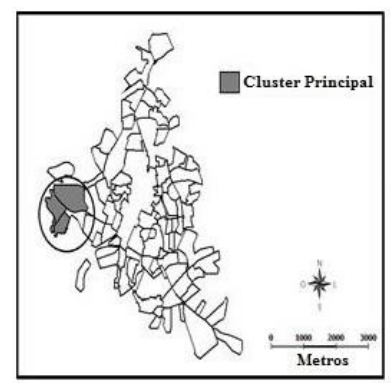

(a)

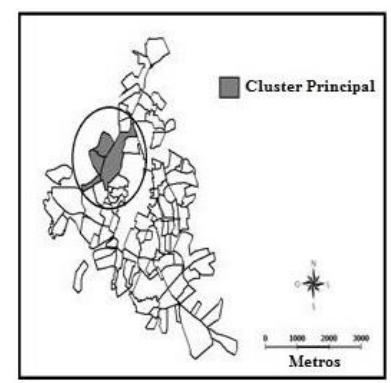

(b)

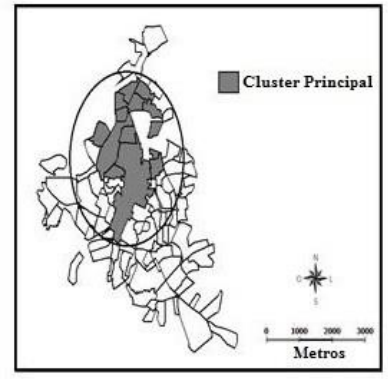

Fonte: Autores.

Uma comparação, entre os clusters que foram detectados no Índice Local de Moran e a análise de varredura espacial, revelou que, em 2007, os bairros que coincidiram com dependência espacial foram os de IDs 202 e 21. Em 2008, foram os de IDs 84, 179, e 183. Em 2010, foram os de IDs 83, 81, 84, 179 e 28. Os bairros que apareceram em ambas as análises merecem mais atenção, uma vez que essas áreas representam potenciais clusters onde a dengue persiste. 
Por meio dos resultados da análise espaço-tempo, também utilizando o programa SaTScan, o cluster mais provável (com estatística do teste de 81,8 ) que foi detectado inclui as seguintes IDs: 202, 21 e 201, de acordo com a Figura 4 (a).

Curiosamente, a análise espaço-tempo revelou que o cluster mais provável que foi detectado durante os quatro anos correspondeu precisamente com o cluster que foi detectado com a análise de varredura espacial para o ano de 2007. Essa situação parece ser contraditória, pois o maior número de casos de dengue foi verificado no ano de 2010. No entanto, o fator determinante para essa análise é que os casos de dengue em 2010 estão distribuídos mais uniformemente entre os bairros, situação esta que não ocorre em 2007. Esse método de detecção de clusters tem um poder baixo para um grande número de pequenos clusters que estão espacialmente distribuídos.

Os bairros que registraram dengue durante os quatro anos analisados foram os de IDs 171 e 84; em outras palavras, a doença foi persistente nessas áreas.

Pode-se verificar que o resultado da estatística Scan Espaço-Tempo não difere muito da primeira análise exploratória dos dados correspondente ao mapa temático do ano de 2007. Nota-se que apenas a ID 202 não aparece como a área de maior número de casos da doença.

\section{CONCLUSÃO}

A partir da pesquisa realizada, foi possível avaliar diferentes técnicas para o estudo de casos de dengue. A construção de mapas temáticos, por exemplo, oferece apenas uma visão exploratória dos dados. Por outro lado, os mapas de incidência possibilitam uma visão da proporção da doença comparada com o tamanho da população de cada bairro. O Índice Global de Moran se mostrou um método mais geral, indicando, apenas, se a região toda analisada tem ou não dependência espacial, ao contrário do Índice Local de Moran, que já oferece uma abordagem mais detalhada da detecção de clusters, embora o grau de detalhamento seja muito sensível. A estatística Scan puramente espacial, como as demais técnicas referidas anteriormente requer uma análise para cada um dos anos estudados. A vantagem dessa análise está no fato de que o usuário pode escolher com qual modelo irá trabalhar, entre as distribuições: Poisson, Bernoulli, Multinomial, Ordinal, Exponencial e Normal. Por outro lado, a estatística Scan Espaço-Tempo possibilita uma análise bem mais dinâmica, em que todos os anos podem ser relacionados de uma vez só, além de permitir uma fundamentação estatística para a validação dos clusters. Dessa forma, a estatística Scan Espaço-temporal se mostrou a técnica mais eficiente e com vantagens sugestivas em relação às demais técnicas analisadas.

\section{AGRADECIMENTOS}

À Coordenação de Aperfeiçoamento de Pessoal de Nível Superior (Capes), pelo 
financiamento da bolsa de estudos.

À Secretaria de Saúde, ao $8^{\circ}$ Batalhão de Polícia Militar, a MDA Pesquisas e à Universidade Federal de Lavras, pelo fornecimento dos dados, fundamentais para o desenvolvimento deste trabalho.

\section{REFERÊNCIAS}

ALMEIDA, M. C. M. et al. Dinâmica intra-urbana das epidemias de dengue em Belo Horizonte, Minas Gerais, Brasil, 1996-2002. Cad Saúde Pública, v. 24, n. 10, p. 238595, 2008.

ANSELIN, L. Local Indicator of Spatial Association - LISA. Geographical Analisys, v. 27, n. 3, p. 93-115, 1995.

ASSUNÇÃO, R. M. Estatística espacial com aplicações em epidemiologia, economia e sociologia. 1. ed. São Carlos: Associação Brasileira de Estatística, v. 1, p. 131, 2000.

BAILEY, T. C.; GATRELL, A. C. Interactive spatial data analysis. 1st ed. Essex, Longman Scientific and Technical, 1995.

BEATO FILHO, C. C. et al. Conglomerados de homicídios e o tráfico de drogas em Belo Horizonte, Minas Gerais, Brasil, de 1995 a 1999. Cad Saúde Pública, v. 17, n. 5 p. 1163-1171, 2001.

BESSA JÚNIOR, F. N. et al. Spatial distribution of dengue disease in municipality of Mossoró, Rio Grande do Norte, using the Geographic Information System. Revista Brasileira de Epidemiologia, v.16, n.3, p.603-610, set., 2013.

CÂMARA, G. et al. Análise espacial de dados geográficos. Brasília, EMBRAPA, 2004. Disponível em: <:http://www.dpi.inpe.br/gilberto/livro/analise/>. Acesso em: 10 jan. 2017.

FLAUZINO, R. F. et al. Heterogeneidade espacial da dengue em estudos locais, Niterói, RJ. Rev Saúde Pública, v.43, n.6, p.1035-1043, 2009.

GALLI, B.; CHIARAVALLOTI NETO, F. Modelo de risco tempo-espacial para identificação de áreas de risco para ocorrência de dengue. Rev Saúde Pública, v. 42, n. 4, p. 656-663, 2008.

GRIFFITH, D. A.; PAELINCK, J. H. P. Non-standard spatial statistics and spatial econometrics. Advances in Geographic Information Science, v. 1, 2011.

HJALMARS, U. et al. Childhood leukemia in Sweden: using GIS and a spatial scan statistic for cluster detection. Statistics in Medicine, v.15, p.707-715, 1996.

IBGE - Instituto Brasileiro de Geografia e Estatística. Disponível em:

$<$ :http://cidades.ibge.gov.br/xtras/perfil.php?codmun=313820>. Acesso em: 12 jan.

2017. 
JEEFOO, P., TRIPATHI, N. K.; SOURIS, M. Spatio-temporal diffusion pattern and hotspot detection of dengue in Chachoengsao Province, Thailand. International Journal of Environmental Research and Public Health, v.8, n.1, p.51-74, 2011.

KULLDORFF, M. Prospective time periodic geographical disease surveillance using a scan statistic. Journal of the Royal Statistical Society: Series A (Statistics in Society), v.164, n.1, p.61-72, 2001.

KULLDORFF, M. et al. A space-time permutation scan statistic for disease outbreak detection. PLoS Medicine, v.2, n.3, p.e59, 2005.

KULLDORFF, M. et al. Evaluating cluster alarms: a spatio-temporal scan statistic and brain cancer in Los Alamos, New Mexico. American Journal of Public Health, v.88, n.9, p.1377-1380, 1998.

KULLDORFF, M. et al.. An elliptic spatial scan statistic. Statistics in Medicine, v. 25, n. 22, p. 3929-3943, 2006.

KULLDORFF, M., FEUER, E. J.; MILLER, B. A., Freedman LS. Breast cancer clusters in the Northeast United States: a geographic analysis. American Journal of Epidemiology, v.146, n.2, p.161-170, 1997.

KULLDORFF, M.; NAGARWALLA, N. Spatial disease clusters: detection and inference. Statistics in Medicine, v.14, n.8, p.799-810, 1995.

LAWSON, A. B. Statistical Methods in spatial epidemiology. Sussex: John Wiley \& Sons, 2001.

LEITE, M. E.; ABREU, K. K. R. C. Sistema de Informação Geográfica aplicado à distribuição do caso de dengue na microrregião de Pirapora - MG. Hygeia, Revista Brasileira de Geografia Médica e da Saúde, v.5, n.9, p.63-76, 2009.

LUCENA, S. E. F.; MORAES, R. M. Detecção de agrupamentos espaço-temporais para identificação de áreas de risco de homicídios por arma branca em João Pessoa, PB. Boletim de Ciências Geodésicas, v. 18, n. 4, p. 605-623, 2012.

MACHADO, J. P., OLIVEIRA, R. M.; SANTOS, R. S. Análise espacial da ocorrência de dengue e condições de vida na cidade de Nova Iguaçu, Estado do Rio de Janeiro, Brasil. Cad Saúde Pública, v. 25, n. 5, p.1025-1034, 2009.

MONDINI, A. et al Spatial analysis of dengue transmission in a medium-sized city in Brazil. Rev Saúde Pública, v.39, n.3, p.444-451, 2005.

MONDINI, A.; CHIARAVALLOTI NETO, F. Variáveis socioeconômicas e a transmissão de dengue. Rev Saúde Pública, v. 41, n. 6, p. 923-930, 2007.

MORRISON, A. C. et al. Exploratory spatio-temporal analysis of reported dengue cases during an outbreak in Florida, Puerto Rico, 1991-1992. American Journal of Tropical Medicine and Hygiene, v.58, n.3, p.287-298, 1998.

ROJAS, L. I., BARCELLOS, C.; PEITER, P. Utilização de mapas no campo da epidemiologia no Brasil: reflexões sobre trabalhos apresentados no IV congresso brasileiro de epidemiologia. Informe Epidemiológico do SUS, v. 8, n. 2, p. 27-35, 1999. 
ROTELA, C. et al. Space-time analysis of the dengue spreading dynamics in the 2004 Tartagal outbreak, Northern Argentina. Acta Tropica, v.103, n.1, p.1-13, 2007.

SCHMIDT, W. P. et al. Population density, water supply, and the risk of dengue fever in Vietnam: cohort study and spatial analysis. PLoS Medicine, v.8, n.8, p.e1001082, aug., 2011.

TEIXEIRA, T. R. A.; CRUZ, O. G. Spatial modeling of dengue and socio-environmental indicators in the city of Rio de Janeiro, Brazil. Cad Saúde Pública, v. 27, n. 3, p. 591602, 2011.

TEIXEIRA, T. R. A.; MEDRONHO, R. A. Indicadores sócio-demográficos e a epidemia de dengue em 2002 no Estado do Rio de Janeiro, Brasil. Cad Saúde Pública, v. 24, n. 9, p. 2160-2170, 2008.

TRAN, A. et al. Dengue spatial and temporal patterns, French Guiana, 2001.

Emerging Infectious Diseases, v.10, n.4, p.615-621, 2004. 